\title{
Eigenvalue comparisons in Steklov eigenvalue problem and some other eigenvalue estimates
}

\author{
Yan Zhao ${ }^{1}$, Chuanxi $\mathrm{Wu}^{1}$, Jing Mao ${ }^{1,2, *}$, Feng $\mathrm{Du}^{3}$ \\ ${ }^{1}$ Faculty of Mathematics and Statistics, \\ Key Laboratory of Applied Mathematics of Hubei Province, \\ Hubei University, Wuhan 430062, China \\ 2 Department of Mathematics, Instituto Superior Técnico, University of Lisbon, \\ Av. Rovisco Pais, 1049-001 Lisbon, Portugal \\ Emails: jiner120@163.com,jiner120@tom.com \\ ${ }^{3}$ School of Mathematics and Physics Science, \\ Jingchu University of Technology, Jingmen, 448000, China
}

\begin{abstract}
In this paper, two interesting eigenvalue comparison theorems for the first non-zero Steklov eigenvalue of the Laplacian have been established for manifolds with radial sectional curvature bounded from above. Besides, sharper bounds for the first non-zero eigenvalue of the Wentzell eigenvalue problem of the weighted Laplacian, which can be seen as a natural generalization of the classical Steklov eigenvalue problem, have been obtained.
\end{abstract}

\section{Introduction}

Let $(M, g)$ be an $n$-dimensional $(n \geq 2)$ complete Riemannian manifold with the Riemannian metric $g$, and let $\Omega \subseteq M$ be a compact domain with boundary $\partial \Omega$. The so-called Steklov eigenvalue problem is actually to find a solution of the following system

$$
\begin{cases}\Delta \varphi=0 & \text { in } \Omega, \\ \frac{\partial \varphi}{\partial \vec{\eta}}=v \varphi & \text { on } \partial \Omega,\end{cases}
$$

where $\Delta$ is the Laplacian on $M$ associated with the metric $g, \vec{\eta}$ is the unit outward normal vector field of the boundary $\partial \Omega$, and $v$ is a real number called the eigenvalue of this problem. There are infinitely many real numbers $v$ satisfying the system (1.1) and can be listed increasingly as a sequence tending to the infinity. Clearly, the first Steklov eigenvalue of the problem (1.1) is zero

${ }^{*}$ Cofresponding author MSC 2010: 35P15, 53C20.

Key Words: Steklov eigenvalue problem, Laplacian, eigenvalues, spherically symmetric manifolds, Wentzell eigenvalue problem. 
with the constant function as its eigenfunction. By the variational principle, it is not difficult to get the first non-zero Steklov eigenvalue $v_{1}(\Omega)$ is characterized by

$$
v_{1}(\Omega)=\min _{\int_{\partial \Omega} u=0} \frac{\int_{\Omega}|\nabla u|^{2}}{\int_{\partial \Omega} u^{2}},
$$

where $\nabla$ is the gradient operator on $\Omega \subseteq M$, and $u \in W^{1,2}(\Omega)$, the completion of the set of smooth functions under the Sobolev norm $\|u\|_{1,2}=\int_{\Omega} u^{2}+\int_{\Omega}|\nabla u|^{2}$. The problem (1.1) was introduced by Steklov [25] with the physical background as follows: the function $\varphi$ denotes the steady state temperature on $\Omega$ such that the flux on $\partial \Omega$ is proportional to the temperature. Since the set of eigenvalues for the Steklov eigenvalue problem is the same as the set of eigenvalues of the well-known Dirichlet-to-Neumann map, the problem (1.1) has important influence in the study of conductivity and harmonic analysis, which was initially studied by Calderón [5]. Anyway, Escobar [11] showed that the study of (1.1) is also important in the problem of conformal deformation of a Riemannian metric on manifolds with boundary.

By (1.2), it is easy to get the following Sobolev trace inequality

$$
\int_{\partial \Omega}\left|u-u_{0}\right|^{2} \leq \frac{1}{v_{1}(\Omega)} \int_{\Omega}|\nabla u|^{2}
$$

where $u_{0}$ is the mean value of the function $u$ when restricted to the boundary. This inequality makes an important role in the study of existence and regularity of solutions of some boundary value problems.

In order to state our main conclusions below clearly, here we would like to introduce some basic notions, which have been introduced in [16, 20, 21, 22] already. Besides, in the sequel, for convenience, we will drop the integral measures for all integrals except it is necessary.

\subsection{Basic notions}

As before, let $(M, g)$ be an $n$-dimensional $(n \geq 2)$ complete Riemannian manifold with the metric $g$, and $\nabla$ be the gradient operator. For a point $p \in M$, one can set up a geodesic polar coordinates $(t, \xi)$ around this point $p$, where $\xi \in S_{p}^{n-1} \subseteq T_{p} M$ is a unit vector of the unit sphere $S_{p}^{n-1}$ with center $p$ in the tangent space $T_{p} M$. Let $\mathscr{D}_{p}$, a star shaped set of $T_{p} M$, and $d_{\xi}$ be defined by

$$
\mathscr{D}_{p}=\left\{t \xi \mid 0 \leq t<d \xi, \xi \in S_{p}^{n-1}\right\}
$$

and

$d_{\xi}=d_{\xi}(p):=\sup \left\{t>0 \mid \gamma_{\xi}(s):=\exp _{p}(s \xi)\right.$ is the unique minimal geodesic joining $p$ and $\left.\gamma_{\xi}(t)\right\}$

respectively. Then $\exp _{p}: \mathscr{D}_{p} \rightarrow M \backslash C u t(p)$ gives a diffeomorphism from $\mathscr{D}_{p}$ onto the open set $M \backslash C u t(p)$, with $C u t(p)$ the cut locus of $p$. For $\zeta \in \xi^{\perp}$, one can define the path of linear transformations $\mathbb{A}(t, \xi): \xi^{\perp} \rightarrow \xi^{\perp}$ by

$$
\mathbb{A}(t, \xi) \zeta=\left(\tau_{t}\right)^{-1} Y(t)
$$


with $\xi^{\perp}$ the orthogonal complement of $\{\mathbb{R} \xi\}$ in $T_{p} M$, where $\tau_{t}: T_{p} M \rightarrow T_{\exp _{p}(t \xi)} M$ is the parallel translation along the geodesic $\gamma_{\xi}(t)$ with $\gamma^{\prime}(0)=\xi$, and $Y(t)$ is the Jacobi field along $\gamma_{\xi}$ satisfying $Y(0)=0,\left(\nabla_{t} Y\right)(0)=\zeta$. Moreover, set

$$
\mathscr{R}(t) \zeta=\left(\tau_{t}\right)^{-1} R\left(\gamma_{\xi}^{\prime}(t), \tau_{t} \zeta\right) \gamma_{\xi}^{\prime}(t)
$$

where the curvature tensor $R(X, Y) Z$ is defined by $R(X, Y) Z=-\left[\nabla_{X}, \nabla_{Y}\right] Z+\nabla_{[X, Y]} Z$. Then $\mathscr{R}(t)$ is a self-adjoint operator on $\xi^{\perp}$, whose trace is the radial Ricci tensor

$$
\operatorname{Ric}_{\gamma_{\xi}(t)}\left(\gamma_{\xi}^{\prime}(t), \gamma_{\xi}^{\prime}(t)\right)
$$

Clearly, the map $\mathbb{A}(t, \xi)$ satisfies the Jacobi equation $\mathbb{A}^{\prime \prime}+\mathscr{R} \mathbb{A}=0$ with initial conditions $\mathbb{A}(0, \xi)=$ $0, \mathbb{A}^{\prime}(0, \xi)=I$, and by Gauss's lemma, the Riemannian metric of $M$ can be expressed by

$$
d s^{2}\left(\exp _{p}(t \xi)\right)=d t^{2}+|\mathbb{A}(t, \xi) d \xi|^{2}
$$

on the set $\exp _{p}\left(\mathscr{D}_{p}\right)$. Consider the metric components $g_{i j}(t, \xi), i, j \geq 1$, in a coordinate system $\left\{t, \xi_{a}\right\}$ formed by fixing an orthonormal basis $\left\{\zeta_{a}, a \geq 2\right\}$ of $\xi^{\perp}=T_{\xi} S_{p}^{n-1}$, and extending it to a local frame $\left\{\xi_{a}, a \geq 2\right\}$ of $S_{p}^{n-1}$. Define a function $J>0$ on $\mathscr{D}_{p}$ by

$$
J^{n-1}=\sqrt{|g|}:=\sqrt{\operatorname{det}\left[g_{i j}\right]} .
$$

Since $\tau_{t}: S_{p}^{n-1} \rightarrow S_{\gamma_{\xi}(t)}^{n-1}$ is an isometry, we have

$$
g\left(d\left(\exp _{p}\right)_{t}\left(t \zeta_{a}\right), d\left(\exp _{p}\right)_{t}\left(t \zeta_{b}\right)\right)=g\left(\mathbb{A}(t, \xi)\left(\zeta_{a}\right), \mathbb{A}(t, \xi)\left(\zeta_{b}\right)\right)
$$

and

$$
\sqrt{|g|}=\operatorname{det} \mathbb{A}(t, \xi) \text {. }
$$

So, by (1.3), the volume $V(B(p, r))$ of the geodesic ball $B(p, r)$ on $M$ is given by

$$
V(B(p, r))=\int_{S_{p}^{n-1}} \int_{0}^{\min \left\{r, d_{\xi}\right\}} \sqrt{|g|} d t d \sigma=\int_{S_{p}^{n-1}}\left(\int_{0}^{\min \left\{r, d_{\xi}\right\}} \operatorname{det}(\mathbb{A}(t, \xi)) d t\right) d \sigma,
$$

where $d \sigma$ denotes the $(n-1)$-dimensional volume element on $\mathbb{S}^{n-1} \equiv S_{p}^{n-1} \subseteq T_{p} M$. The injectivity $\operatorname{radius} \operatorname{inj}(p)$ at $p$ satisfies

$$
\operatorname{inj}(p):=d(p, C u t(p))=\min _{\xi} d \xi
$$

In general, we have $B(p, \operatorname{inj}(p)) \subseteq M \backslash C u t(p)$. Besides, for $r<\operatorname{inj}(p)$, by (11.4) we can obtain

$$
V(B(p, r))=\int_{0}^{r} \int_{S_{p}^{n-1}} \operatorname{det}(\mathbb{A}(t, \xi)) d \sigma d t .
$$

Denote by $r(x)=d(x, p)$ the intrinsic distance to the point $p \in M$. Then, by the definition of a non-zero tangent vector "radial" to a prescribed point on a manifold given in the first page of [19], we know that for $x \in M \backslash(C u t(p) \cup p)$ the unit vector field

$$
v_{x}:=\nabla r(x)
$$

is the radial unit tangent vector at $x$. This is because for any $\xi \in S_{p}^{n-1}$ and $t_{0}>0$, we have $\nabla r\left(\gamma_{\xi}\left(t_{0}\right)\right)=\gamma_{\xi}^{\prime}\left(t_{0}\right)$ when the point $\gamma_{\xi}\left(t_{0}\right)=\exp _{p}\left(t_{0} \xi\right)$ is away from the cut locus of $p$.

We need the following concepts. 
Definition 1.1. ([16, 20, 21]) Given a continuous function $k:[0, l) \rightarrow \mathbb{R}$, we say that $M$ has a radial Ricci curvature lower bound $(n-1) k$ along any unit-speed minimizing geodesic starting from a point $p \in M$ if

$$
\operatorname{Ric}\left(v_{x}, v_{x}\right) \geq(n-1) k(r(x)), \quad \forall x \in M \backslash(\operatorname{Cut}(p) \cup\{p\}),
$$

where Ric is the Ricci curvature of $M$.

Definition 1.2. ([16, 20, 21]) Given a continuous function $k:[0, l) \rightarrow \mathbb{R}$, we say that $M$ has a radial sectional curvature upper bound $k$ along any unit-speed minimizing geodesic starting from a point $p \in M$ if

$$
\mathscr{K}\left(v_{x}, V\right) \leq k(r(x)), \quad \forall x \in M \backslash(C u t(p) \cup\{p\}),
$$

where $V \perp v_{x}, V \in S_{x}^{n-1} \subseteq T_{x} M$, and $\mathscr{K}\left(v_{x}, V\right)$ is the sectional curvature of the plane spanned by $v_{x}$ and $V$.

Remark 1.3. Since $r(x)=d(p, x)=t$ and $\left.\frac{d}{d t}\right|_{x}=\nabla r(x)=v_{x}$, we know that the inequalities (1.5) and (1.6) become $\operatorname{Ric}\left(\frac{d}{d t}, \frac{d}{d t}\right) \geq(n-1) k(t)$ and $\mathscr{K}\left(\frac{d}{d t}, V\right) \leq k(t)$, respectively. This fact has been pointed out in [16, Remark 2.4] or [20, Remark 2.1.5]. Besides, for convenience, if a manifold satisfies (1.5) (resp., (1.6)), then we say that $M$ has a radial Ricci curvature lower bound w.r.t. a point $p$ (resp., a radial sectional curvature upper bound w.r.t. a point $p$ ), that is to say, its radial Ricci curvature is bounded from below w.r.t. $p$ (resp., radial sectional curvature is bounded from above w.r.t. p). As also pointed out in [16, page 706] or [21, page 378], for a given complete Riemannian $n$-manifold $(M, g)$, one can define

$$
k_{-}(p, t):=\min _{\left\{\xi \mid(t, \xi) \in \overline{\mathbb{D}}_{p}\right\}} \frac{\operatorname{Ric}_{\gamma_{\xi}(t)}\left(\left.\frac{d}{d t}\right|_{\exp _{p}(t \xi)},\left.\frac{d}{d t}\right|_{\exp _{p}(t \xi)}\right.}{n-1}, \quad 0 \leq t<l(p):=\max _{\xi} d_{\xi}
$$

and

$$
k_{+}(p, t):=\max _{\left\{(\xi, V)\left|\gamma_{\xi}^{\prime}(t) \perp V,\right| V \mid=1\right\}} \mathscr{K}_{\gamma_{\xi}(t)}\left(\left.\frac{d}{d t}\right|_{\exp _{p}(t \xi)}, V\right), \quad 0 \leq t<\operatorname{inj}(p),
$$

where $\mathbb{D}_{p}:=\left\{(t, \xi) \in[0, \infty) \times S_{p}^{n-1} \mid 0 \leq t<d_{\xi}\right\}$ with closure $\overline{\mathbb{D}}_{p}=\left\{(t, \xi) \in[0, \infty) \times S_{p}^{n-1} \mid 0 \leq\right.$ $\left.t \leq d_{\xi}\right\}$, and, by applying the uniform continuity of continuous functions on compact sets, for a bounded domain $\Omega \subseteq M$, one can always find optimally continuous bounds $k_{ \pm}(p, t)$ for the radial sectional and Ricci curvatures w.r.t. some point $p \in \Omega$.

We need the following notion of spherically symmetric manifolds.

Definition 1.4. ([16, 20, 21] $]) A$ domain $\Omega=\exp _{p}\left([0, l) \times S_{p}^{n-1}\right) \subset M \backslash C u t(p)$, with $l<\operatorname{inj}(p)$, is said to be spherically symmetric with respect to a point $p \in \Omega$, if the matrix $\mathbb{A}(t, \xi)$ satisfies $\mathbb{A}(t, \xi)=f(t) \mathrm{I}_{(n-1) \times(n-1)}$, for a function $f \in C^{2}([0, l]), l \in(0, \infty]$ with $f(0)=0, f^{\prime}(0)=1$, $f \mid(0, l)>0$, where $\mathrm{I}_{(n-1) \times(n-1)}$ represents the $(n-1) \times(n-1)$ identity matrix. 
By (1.3), on the set $\Omega$ given in Definition 1.4 the Riemannian metric of $M$ can be expressed by

$$
d s^{2}\left(\exp _{p}(t \xi)\right)=d t^{2}+f^{2}(t)|d \xi|^{2}, \quad \xi \in S_{p}^{n-1}, \quad 0 \leq t<l
$$

with $|d \xi|^{2}$ the round metric on the unit sphere $\mathbb{S}^{n-1} \subseteq \mathbb{R}^{n}$. Spherically symmetric manifolds were named as generalized space forms by Katz and Kondo [19], and a standard model for such manifolds is given by the quotient manifold of the warped product $[0, l) \times_{f} \mathbb{S}^{n-1}$ equipped with the metric (1.7), and all pairs $(0, \xi)$ are identified with a single point $p$, where $f$ satisfies the conditions in Definition 1.4, and is called the warping function. A space form with constant curvature $k$ is also a spherically symmetric manifold, and in this special case we have

$$
f(t)=\left\{\begin{array}{lll}
\frac{\sin \sqrt{k} t}{\sqrt{k}}, & l=\frac{\pi}{\sqrt{k}} & k>0 \\
t, & l=+\infty & k=0 \\
\frac{\sinh \sqrt{-k} t}{\sqrt{-k}}, & l=+\infty & k<0 .
\end{array}\right.
$$

\subsection{The statement of main conclusions}

In [16], Freitas, Mao and Salavessa proved that for $n$-dimensional $(n \geq 2)$ complete Riemannian manifold $(M, g)$ having a radial Ricci curvature lower bound $(n-1) k(t)$ w.r.t. a point $p \in M$, the first Dirichlet eigenvalue $\lambda_{1}(B(p, r))$ of the Laplacian on $B(p, r)$ satisfies

$$
\lambda_{1}(B(p, r)) \leq \lambda_{1}\left(\mathscr{B}_{n}\left(p^{-}, r\right)\right),
$$

where $\lambda_{1}\left(\mathscr{B}_{n}\left(p^{-}, r\right)\right)$ is the first Dirichlet eigenvalue of the Laplacian on the geodesic ball $\mathscr{B}_{n}\left(p^{-}, r\right)$, with the center $p^{-}$and radius $r$, of the spherically symmetric $n$-manifold $M^{-}=[0, l) \times_{f} \mathbb{S}^{n-1}$ with the base point $p^{-}$and the warping function $f$ determined by

$$
\left\{\begin{array}{l}
f^{\prime \prime}(t)+k(t) f(t)=0 \\
f(0)=0, f^{\prime}(0)=1
\end{array} \quad \text { on }(0, l),\right.
$$

Equality in (1.8) holds if and only if $B(p, r)$ is isometric to $\mathscr{B}_{n}\left(p^{-}, r\right)$. They also proved that if $M$ has a radial sectional curvature upper bound $(n-1) k(t)$ w.r.t. a point $p \in M$, then for $r<\operatorname{inj}(p)$,

$$
\lambda_{1}(B(p, r)) \geq \lambda_{1}\left(\mathscr{B}_{n}\left(p^{+}, r\right)\right)
$$

holds, where $\lambda_{1}\left(\mathscr{B}_{n}\left(p^{+}, r\right)\right)$ is the first Dirichlet eigenvalue of the Laplacian on the geodesic ball $\mathscr{B}_{n}\left(p^{+}, r\right)$, with the center $p^{+}$and radius $r$, of the spherically symmetric $n$-manifold $M^{+}=[0, l) \times_{f}$ $\mathbb{S}^{n-1}$ with the base point $p^{+}$and $f$ determined by (1.9). Equality in (1.10) holds if and only if $B(p, r)$ is isometric to $\mathscr{B}_{n}\left(p^{+}, r\right)$. Clearly, the model spaces, i.e., spherically symmetric manifolds, are determined by the curvature bounds. These eigenvalue estimates improve the classical Cheng's eigenvalue comparison theorems [6, 7], whose model spaces are space forms of constant curvature, a lot in the spectral geometry. Besides, Freitas, Mao and Salavessa [16, Section 6] have used interesting examples (i.e., torus, elliptic paraboloid, saddle) to intuitively and numerically show that their comparisons are better than those of Cheng's. The eigenvalue comparison (1.8) has been already extended to the case of nonlinear $p$-Laplacian $(1<p<\infty)$ - see Mao [21, Theorem 3.2] for the detail. 
It is interesting to know whether the system (1.9) has a long-time existence solution (i.e., a positive solution on $(0, \infty)$ and in this case $l=\infty)$ or not. This has close relationship with the oscillation theory of ordinary differential equations (ODEs for short). Clearly, by Sturm-Picone comparison theorem, one can easily get that $l=\infty$ if $k(t) \leq 0$, while $l<\infty$ if $k(t) \geq \alpha$ for some positive constant $\alpha>0$. Readers can check [20, Sect. 2.6, Chapt. 2] for the details about the general restrictions on $k(t)$ to get the existence or non-existence of positive solution to (1.9) on $(0, \infty)$.

By using spherically symmetric manifolds as the model spaces also, we can get the following eigenvalue comparisons for the first non-zero Steklov eigenvalue for the system (1.1) with $\Omega$ chosen as geodesic balls.

Theorem 1.5. For a given $n$-dimensional $(n=2,3)$ complete Riemannian manifold $(M, g)$ having a radial sectional curvature upper bound $k(t)$ w.r.t. $p$, where, as before, $t:=d(p, \cdot)$ represents the distance to the point $p \in M$, and $k(t)$ is a continuous function w.r.t. $t$, we have

$$
v_{1}(B(p, r)) \leq v_{1}\left(\mathscr{B}_{n}\left(p^{+}, r\right)\right)
$$

where $r<\operatorname{inj}(p)$, and $\mathscr{B}_{n}\left(p^{+}, r\right)$ is the geodesic ball, with the center $p^{+}$and radius $r$, of the spherically symmetric n-manifold $M^{+}=[0, l) \times_{f} \mathbb{S}^{n-1}$ with the base point $p^{+}$and the warping function $f$ determined by (1.9). Equality holds if and only if $B(p, r)$ is isometric to $\mathscr{B}_{n}\left(p^{+}, r\right)$.

For the higher dimensional case, we have the following.

Theorem 1.6. Assume that same notations have the same meaning as in Theorem 1.5, For a given $n$-dimensional $(n \geq 2)$ complete Riemannian manifold $(M, g)$ having a radial sectional curvature upper bound $k(t)$ w.r.t. $p$, if the first non-zero closed eigenvalues of the Laplacian on the boundary satisfy

$$
\lambda_{1}^{c}(\partial B(p, r)) \leq \lambda_{1}^{c}\left(\partial \mathscr{B}_{n}\left(p^{+}, r\right)\right)
$$

with $r<\operatorname{inj}(p)$, then we have

$$
v_{1}(B(p, r)) \leq v_{1}\left(\mathscr{B}_{n}\left(p^{+}, r\right)\right) .
$$

Equality holds if and only if $B(p, r)$ is isometric to $\mathscr{B}_{n}\left(p^{+}, r\right)$.

Remark 1.7. (1) For the purse of letting readers understand the assumption (1.11) clearly, here we would like to give a brief introduction to the closed eigenvalue problem of the Laplacian. For an open, bounded, connected domain $D \subset M$, without boundary, on a given Riemannian $n$-manifold $M$, the so-called closed eigenvalue problem of the Laplacian on $D$ is actually to find a nontrivial solution to $\Delta u+\lambda^{c} u=0$ in $D, u \in W^{1,2}(D)$. It is well-known that $-\Delta$ only has discrete spectrum and all the elements in the spectrum (i.e., eigenvalues) can be listed non-decreasingly into a sequence tending to infinity, i.e., $0=\lambda_{0}^{c}(D)<\lambda_{1}^{c}(D) \leq \lambda_{2}^{c}(D) \leq \lambda_{3}^{c}(D) \leq \cdots \uparrow \infty$. For each eigenvalue $\lambda^{c}$, the corresponding $u$ to the equation $\Delta u+\lambda^{c} u=0$ is called the eigenfunction of $\lambda^{c}$. Clearly, the eigenfunction of $\lambda_{0}^{c}(D)=0$ is a constant function over $D$. Besides, by the variational principle, one knows that the first non-zero closed eigenvalue $\lambda_{1}^{c}(D)$ can be characterized as follows

$$
\lambda_{1}^{c}(D)=\inf \left\{\frac{\int_{D}|\nabla u|^{2}}{\int_{D} u^{2}} \mid u \in W^{1,2}(D), u \neq 0, \int_{D} u=0\right\} .
$$


(2) The assumption (1.11) is always true for lower dimensions $n=2,3$ (see pages 16-17 for details), so Theorem 1.5 looks much more natural and better than Theorem 1.6.

(3) As pointed out by Escobar [13, page 145], the restraint on the injectivity radius is necessary. In fact, Escobar constructed a geodesic ball $B(p, r)$, with $r>\operatorname{inj}(p)$, in a flat two-dimensional torus such that $v_{1}(B(p, r))>\frac{1}{r}$, which is strictly bigger than the first non-zero Steklov eigenvalue $\frac{1}{r}$ of the Euclidean 2-ball with radius $r$.

(4) Escobar [12, pages 109-111] used non-simply-connected annuli as the example to explain the fundamental difference between the Dirichlet eigenvalue problem and the Steklov eigenvalue problem of the Laplacian, which implies that the research experience on the Dirichlet eigenvalue problem might not be used in the study of the Steklov eigenvalue problem directly.

(5) Clearly, one can get Escobar's main conclusions [13, Theorems 1 and 2] by directly applying our Theorems 1.5 and 1.6 twice. Therefore, our conclusions here are sharper than Escobar's and cover them as special cases. Inspired by examples constructed in [16, 20, 21], we would like to use the torus example below to let readers realize the advantage of our comparisons here intuitively.

- Let $\{x, y, z\}$ be the Cartesian coordinates of the Euclidean 3 -space $\mathbb{R}^{3}$. Consider the ring torus $\mathscr{T}$ given by

$$
\left\{\begin{array}{l}
x=(1+\varepsilon \cdot \cos v) \cos u \\
y=\varepsilon \cdot \sin v \\
z=(1+\varepsilon \cdot \cos v) \sin u
\end{array}\right.
$$

with $u, v \in[0,2 \pi)$ and $0<\varepsilon<1$. Clearly, $\mathscr{T}$ can be obtained by rotating the circle $(x-$ $1)^{2}+y^{2}=\varepsilon^{2}$ with respect to the $y$-axis. Denote this circle by $\mathscr{C}$. It is not difficult to know that the Gaussian curvature of $\mathscr{T}$ is given by

$$
K=\frac{\cos v}{\varepsilon(1+\varepsilon \cdot \cos v)}
$$

Without loss of generality, we can choose $\varepsilon=\frac{1}{2}$, and then $K=\frac{4 \cos v}{2+\cos v}, \mathscr{T}$ is explicitly expressed as

$$
\left(1-\sqrt{x^{2}+z^{2}}\right)^{2}+y^{2}=\frac{1}{4}
$$

As shown clearly in [20, Example 2.5.1, Sect. 2.5, Chapt. 2] (or using the method explained in [21, Example 4.4] for finding sharp lower bounds for the Gaussian curvature), I for the geodesic ball $B(p, r)$ on $\mathscr{T}$, where $0 \leq r<\frac{\pi}{2}$, I one can get the following facts:

Case 1. If $p$ is one of those points which are farthest from the $y$-axis, that is, $p$ locates on the circle $C_{1}$ in the $x o z$-plane defined by $x^{2}+z^{2}=9 / 4$. Without loss of generality, we can choose $p$ to be the point $(3 / 2,0,0)$, which implies that $p$ is also on the circle $\mathscr{C}$. Clearly,

\footnotetext{
${ }^{1}$ In the 2-dimensional case, the radial Ricci curvature and the radial sectional curvature degenerate into the Gaussian curvature. Hence, here if one wants to use our conclusion Theorem 1.5 for a given parameterized surface, the only thing needed is finding upper bounds for the Gaussian curvature - see Remark 1.3 for the principle of getting optimal bounds. By Theorem 1.5 , we know that the more sharper upper bounds found, the more shaper upper bounds can be given for $v_{1}(B(p, r))$.

${ }^{2}$ This range of $r$ is used to make sure that $B(p, r)$ is within the cut-locus of $p$, and then Theorem 1.5 can be applied directly. For the detailed reason, please check [20, Example 2.5.1, Sect. 2.5, Chapt. 2] or [21, Example 4.4].
} 
the parameter $v$ satisfies $v=0$ at the point $p$. In this case, the best upper bound for the Gaussian curvature what we can choose is $K_{\text {upper }}^{1}=4 / 3$, which implies the upper bound for $v_{1}(B(p, r))$ given by Theorem 1.5 is the same with the one determined by Escobar's eigenvalue comparison [13, Theorem 1].

Case 2. If $p$ is one of those points which are nearest to the $y$-axis, that is, $p$ locates on the circle $C_{2}$ in the $x o z$-plane defined by $x^{2}+z^{2}=1 / 4$. Without loss of generality, we can choose $p$ to be the point $(1 / 2,0,0)$, which implies $p \in \mathscr{C}$. In this case, at $p, v=\pi$ and the best upper bound for the Gaussian curvature what we can choose is $K_{\text {upper }}^{2}=\frac{4 \cos (\pi-2 t)}{2+\cos (\pi-2 t)}$, $0 \leq t<\frac{\pi}{2}$, where $t:=d(p, \cdot)$ is the Riemannian distance to $p$. Moreover, the model manifold is $M_{2}^{+}:=[0, l) \times_{f_{2}(t)} \mathbb{S}^{1}$ with the base point $p_{2}^{+}$, where $f_{2}(t)$ is the solution to the system (1.9) with $k(t)=K_{\text {upper }}^{2}$. By Theorem 1.5 and Fact 2 in Section 2, we have

$$
v_{1}(B(p, r)) \leq v_{1}\left(\mathscr{B}_{2}\left(p_{2}^{+}, r\right)\right)=\frac{1}{f_{2}(r)}, \quad \text { for any } 0<r<\frac{\pi}{2} .
$$

However, if one wants to use Escobar's conclusion [13, Theorem 1], the best constant upper bound of the Gaussian curvature should be $\widetilde{K}_{\text {upper }}^{2}=\frac{4 \cos 2 r}{-2+\cos 2 r}:=k_{0}$ since the function $\frac{4 \cos (\pi-2 t)}{2+\cos (\pi-2 t)}$ is increasing on $[0, r)$, and, in this setting, the model manifold is $\mathbb{S}^{2}\left(k_{0}\right)$ if $k_{0}>0$, i.e., $\frac{\pi}{4}<r<\frac{\pi}{2}$; $\mathbb{R}^{2}$ if $k_{0}=0$, i.e., $k_{0}=\frac{\pi}{4}$; $\mathbb{H}^{2}\left(k_{0}\right)$ if $k_{0}<0$, i.e., $0 \leq r<\frac{\pi}{4}$, which is actually the 2-dimensional space form with constant curvature $k_{0}$. Since spherically symmetric manifolds are natural generalization of space forms, which leads to the fact that Escobar's model space here is actually the special spherically symmetric surface $\widetilde{M}_{2}^{+}:=[0, \widetilde{l}) \times \widetilde{f}_{2}(t) \mathbb{S}^{1}$ (endowed with a one-point compactification topology if $k_{0}>0$ ), where

$$
\widetilde{f}_{2}(t)=\left\{\begin{array}{lll}
\frac{\sin \sqrt{k_{0}} t}{\sqrt{k_{0}}}, & \tilde{l}=\frac{\pi}{\sqrt{k_{0}}}, & k_{0}>0 \\
t, & \widetilde{l}=+\infty, & k_{0}=0 \\
\frac{\sinh \sqrt{-k_{0}} t}{\sqrt{-k_{0}}}, & \tilde{l}=+\infty, & k_{0}<0
\end{array}\right.
$$

is a solution to the system (1.9) with $k(t)=k_{0}=\widetilde{K}_{\text {upper }}^{2}$. Hence, by using Escobar's conclusion [13, Theorem 1] and Fact 2, one has

$$
v_{1}(B(p, r)) \leq \frac{1}{\widetilde{f}_{2}(r)}, \quad \text { for any } 0<r<\frac{\pi}{2} .
$$

Since $f_{2}(t), \widetilde{f}_{2}(t)$ satisfy the system (1.9) with different curvature upper bounds $K_{\text {upper }}^{2}$ and $\widetilde{K}_{\text {upper }}^{2}$, by using the fact

$$
K_{\text {upper }}^{2}<\widetilde{K}_{\text {upper }}^{2}=k_{0}, \quad 0<t<r<\frac{\pi}{2}
$$

and the Sturm-Picone comparison theorem, we have $f_{2}(r)>\widetilde{f}_{2}(r)$ for any $0<r<\frac{\pi}{2}$, i.e.,

$$
\frac{1}{f_{2}(r)}<\frac{1}{\widetilde{f}_{2}(r)}, \quad \text { for any } 0<r<\frac{\pi}{2},
$$


which implies, in Case 2, our upper bound for $v_{1}(B(p, r))$ given by (1.13) is sharper than the one in (1.14) determined by Escobar's eigenvalue comparison [13, Theorem 1].

Case 3. If $p$ is neither a point on the circle $C_{1}$ nor a point on the circle $C_{2}$. Without loss of generality, we can choose $p$ to be a point, which is different from the points $(3 / 2,0,0)$ and $(1 / 2,0,0)$, on the circle $\mathscr{C}$. Assume $v=\alpha$ at $p$ with $0<\alpha<\pi$ or $\pi<\alpha<2 \pi$. By the symmetry of $\mathscr{T}$ w.r.t. the $x o y$-plane, without loss of the generality, we can assume $0<\alpha<\pi$. In this case, the best upper bound for the Gaussian curvature what we can choose is

$$
K_{\text {upper }}^{3}= \begin{cases}\frac{4 \cos (\alpha-2 t)}{2+\cos (\alpha-2 t)}, & 0 \leq t \leq \frac{\alpha}{2}, \\ \frac{4}{3}, & \frac{\alpha}{2}<t<\frac{\pi}{2} .\end{cases}
$$

If one wants to use Escobar's conclusion [13, Theorem 1], the best constant upper bound of the Gaussian curvature should be

$$
\widetilde{K}_{\text {upper }}^{3}= \begin{cases}\frac{4 \cos (\alpha-2 r)}{2+\cos (\alpha-2 r)}, & 0 \leq t<r \leq \frac{\alpha}{2}, \\ \frac{4}{3}, & \frac{\alpha}{2}<t<r<\frac{\pi}{2} .\end{cases}
$$

Clearly, $K_{\text {upper }}^{3}<\widetilde{K}_{\text {upper }}^{3}$ for $0 \leq t<r \leq \frac{\alpha}{2}$, and $K_{\text {upper }}^{3}=\widetilde{K}_{\text {upper }}^{3}$ for $\frac{\alpha}{2}<t<r<\frac{\pi}{2}$. Using a similar argument to Case 2, we know that for $0 \leq r<\frac{\pi}{2}$, the upper bound for $v_{1}(B(p, r))$ given by Theorem 1.5 is sharper than the one determined by Escobar's eigenvalue comparison [13, Theorem 1].

(6) By [14, Lemma 2.1], we know that for a given complete surface $\Sigma$, which can be parameterized, and a geodesic ball $B(p, r)$, with $r<\operatorname{inj}(p)$, on $\Sigma$, the optimal upper bound for the Gaussian curvature is actually the maximal values of the Gaussian curvature on geodesic circles $C(p, t)$ with center $p$ and radii $0<t<r$, and this optimal upper bound can be computed numerically. This fact tells us that one can use any parameterized complete surface, not only the ring torus example mentioned above, to show the advantage of Theorem 1.5 here.

Now, we would like to introduce our estimates for the first non-zero eigenvalue of the Wentzell eigenvalue problem of the weighted Laplacian, which can be seen as a natural generalization of the classical Steklov eigenvalue problem. However, before that, we need to briefly mention several notions introduced by Bakry and Émery [1]].

Let $(N,\langle\cdot, \cdot\rangle)$ be an $(n+1)$-dimensional compact oriented Riemannian manifold with smooth boundary $\partial N$. The triple $\left(N,\langle\cdot, \cdot\rangle, e^{-\phi} d v\right)$ is called a metric measure space (MMS for short), where $\phi \in C^{\infty}(N)$ is a smooth function defined on $N$, and $d v$ is the volume element. As introduced by Bakry-Émery [1], the weighted Laplacian (or the drifting Laplacian) and the $K$-dimensional Bakry-Émery Ricci curvature can be defined as follows

$$
\Delta_{\phi}=\Delta-\langle\cdot, \nabla \phi\rangle
$$

and

$$
\operatorname{Ric}_{\phi}^{K}=\operatorname{Ric}+\operatorname{Hess} \phi-\frac{d \phi \otimes d \phi}{K-n-1}
$$


where, with the abuse of notations, $\Delta$ and $\nabla$ denote the Laplace and the gradient operators on $N$ respectively, and Hess is the Hessian operator on $N$ associated to the metric $\langle\cdot, \cdot\rangle$. Here $K>n+1$ or $K=n+1$ if $\phi$ is a constant function. When $K=\infty$, one can defined the so-called $\infty$-dimensional Bakry-Émery Ricci curvature (simply, Bakry-Émery Ricci curvature or weighted Ricci curvature) as follows

$$
\operatorname{Ric}_{\phi}=\operatorname{Ric}+\operatorname{Hess} \phi
$$

By abuse of the notation, denote also by $\vec{\eta}$ the outward unit normal vector field along the boundary $\partial N$. Let $i: \partial N \hookrightarrow N$ be the standard inclusion. For $X, Y \in \mathscr{H}(\partial N)$, i.e., the set of tangent vector fields on $\partial N$, the second fundament form $I I$ associated to $\vec{\eta}$ is given by $\left\langle\vec{\eta}, \nabla_{X} Y\right\rangle=I I(X, Y)$, and the mean curvature $H=\frac{1}{n} \operatorname{tr} I I$ of $\partial N$ is actually the average of the trace of the second fundamental form. Naturally, one can define the so-called weighted mean curvature of the inclusion $i$ as $H_{\phi}=$ $H-\frac{1}{n}\langle\vec{\eta}, \nabla \phi\rangle$ on the MMS $\left(N,\langle\cdot, \cdot\rangle, e^{-\phi} d v\right)$ - see, e.g., [17] for this notion.

On the compact MMS $\left(N,\langle\cdot, \cdot\rangle, e^{-\phi} d v\right)$ mentioned above, consider the following eigenvalue problem with the Wentzell-type boundary condition

$$
\begin{cases}\Delta_{\phi} u=0, & \text { in } N \\ -\beta \bar{\Delta}_{\phi} u+\frac{\partial u}{\partial \vec{\eta}}=\tau u, & \text { on } \partial N\end{cases}
$$

where $\bar{\Delta}_{\phi}$ is the weighted Laplacian on the boundary $\partial N$. In fact, (1.15) is called the Wentzell eigenvalue problem of the weighted Laplacian. When $\phi=$ const., i.e., the non-zero constant function, $\Delta_{\phi}$ and $\bar{\Delta}_{\phi}$ degenerate into usual Laplacians $\Delta$ on $N$ and $\bar{\Delta}$ on the boundary $\partial N$ respectively, and (1.15) becomes

$$
\begin{cases}\Delta u=0, & \text { in } N, \\ -\beta \bar{\Delta} u+\frac{\partial u}{\partial \vec{\eta}}=\tau u, & \text { on } \partial N\end{cases}
$$

which, for $\beta \geq 0$, has discrete spectrum and all the eigenvalues in the spectrum can be listed increasingly as follows

$$
0=\tau_{0}<\tau_{1} \leq \tau_{2} \leq \tau_{3} \leq \cdots \uparrow \infty
$$

Recently, some interesting estimates for eigenvalues $\tau_{i}$ of the eigenvalue problem (1.16) have been obtained - see, e.g., [8, 9, 10, 27]. Especially, when $\beta=0$, (1.16) degenerates into the Steklov eigenvalue problem (1.1).

It is not difficult to find out that the weighted version (1.15) with $\beta \geq 0$ also has discrete spectrum and all the eigenvalues in the spectrum can be listed increasingly as follows

$$
0=\tau_{0, \phi}<\tau_{1, \phi} \leq \tau_{2, \phi} \leq \tau_{3, \phi} \leq \cdots \uparrow \infty
$$

Besides, by the variational principle, it is easy to know that the first non-zero eigenvalue $\tau_{1, \phi}$ of the eigenvalue problem (1.15) can be characterized as follows

$$
\tau_{1, \phi}=\min \left\{\frac{\int_{N}|\nabla u|^{2}+\beta \int_{\partial N}|\bar{\nabla} u|^{2}}{\int_{\partial N} u^{2}} \mid u \in W^{1,2}(N), u \neq 0, \int_{\partial N} u=0\right\},
$$


where $\bar{\nabla}$ is the gradient operator on $\partial N$. Here, we would like to point out one thing that all integrations in (I.17) should have weighted volume elements, and for convenience, we have dropped them. For $\tau_{1, \phi}$, we can obtain its lower and sharp upper bounds under suitable assumptions on the $K$-dimensional Bakry-Émery Ricci curvature $\operatorname{Ric}_{\phi}^{K}$, the weighted mean curvature $H_{\phi}$, and the second fundamental form $I I$ of the boundary $\partial N$ - see Theorems 4.1 and 4.2 for details.

The paper is organized as follows. Some preliminary facts will be mentioned in Section 2. Proofs for Theorems 1.5 and 1.6 will be shown carefully in Section 3. In Section 4, estimates for the first non-zero eigenvalue of the Wentzell eigenvalue problem of the weighted Laplacian will be investigated. An open problem will be issued in the last section.

\section{Some useful facts}

In this section, some facts will be mentioned in the purpose of proving the main conclusions of this paper. However, before that, let us recall several useful facts about spherically symmetric manifolds.

By making the radial sectional curvature upper bound assumption, Mao [20, Theorem 2.3.2] (see also [16, Theorem 4.2]) has proven the following Bishop-type volume comparison theorem.

Theorem 2.1. ([16] 20], generalized Bishop's volume comparison theorem II) Assume that $M$ has a radial sectional curvature upper bound $k(t)=-\frac{f^{\prime \prime}(t)}{f(t)}$ w.r.t. $p \in M$ for $t<\alpha \leq \min \left\{\operatorname{inj}_{c}(p), l\right\}$, where in $j_{c}(p)=\inf _{\xi} c_{\xi}$, with $\gamma_{\xi}\left(c_{\xi}\right)$ a first conjugate point along the geodesic $\gamma_{\xi}(t)=\exp _{p}(t \xi)$. Then on $(0, \alpha)$,

$$
\left(\frac{\sqrt{|g|}}{f^{n-1}}\right)^{\prime} \geq 0, \quad \sqrt{|g|}(t, \xi) \geq f^{n-1}(t),
$$

and equality occurs in the first inequality at $t_{0} \in(0, \alpha)$ if and only if

$$
\mathscr{R}=-\frac{f^{\prime \prime}(t)}{f(t)}, \quad \mathbb{A}=f(t) \mathbf{I}_{(n-1) \times(n-1)},
$$

on all of $\left[0, t_{0}\right]$.

There are another three important facts for our model spaces, which will be used later, we would like to list here.

- Fact 1: ([16, page 706], [21, page 379]) By proposition 42 and corollary 43 of chapter 7 in [23] or subsection 3.2.3 of chapter 3 in [24], we know that the radial sectional curvature, and the component of the radial Ricci tensor of the spherically symmetric manifold $M^{*}=$ $[0, l) \times_{f(t)} \mathbb{S}^{n-1}$ with the base point $p$ are given by

$$
\begin{aligned}
& \mathscr{K}\left(V, \frac{d}{d t}\right)=R\left(\frac{d}{d t}, V, \frac{d}{d t}, V\right)=-\frac{f^{\prime \prime}(t)}{f(t)} \quad \text { for } V \in T_{\xi} \mathbb{S}^{n-1},|V|_{g}=1, \\
& \operatorname{Ric}\left(\frac{d}{d t}, \frac{d}{d t}\right)=-(n-1) \frac{f^{\prime \prime}(t)}{f(t)} .
\end{aligned}
$$

Thus, Definition 1.1 (resp., Definition 1.2) is satisfied with equality in (1.5) (resp., (1.6) ) and $k(t)=-f^{\prime \prime}(t) / f(t)$. 
- Fact 2: ([13, Lemma 3]) Let $B_{r}$ be a ball in $\mathbb{R}^{n}$ endowed with a rotationally invariant metric

$$
d t^{2}+f^{2}(t)|d \xi|^{2}
$$

where, as before, $|d \xi|^{2}$ represents the round metric on the unit sphere $\mathbb{S}^{n-1} \subseteq \mathbb{R}^{n}$. The first nonconstant eigenfunction for the Steklov problem on $B_{r}$ has the form

$$
\varphi(t, \xi)=\psi(t) e(\xi)
$$

where $e(\xi)$ satisfies the equation $\Delta e+(n-1) e=0$ on $\mathbb{S}^{n-1}$ and the function $\psi$ satisfies the differential equation

$$
\begin{aligned}
& \frac{1}{f^{n-1}(t)} \frac{d}{d t}\left(f^{n-1}(t) \frac{d}{d t} \psi(t)\right)-\frac{(n-1) \psi(t)}{f^{2}(t)}=0 \quad \text { in }(0, r), \\
& \psi^{\prime}(r)=v_{1}\left(B_{r}\right) \psi(r), \quad \psi(0)=0 .
\end{aligned}
$$

- Fact 3: ([13, Proposition 4]) Let $B_{r}$ be a two-dimensional ball in $\mathbb{R}^{2}$ endowed with a rotationally invariant metric

$$
d t^{2}+f^{2}(t)|d \xi|^{2}
$$

Then the first nonzero Steklov eigenvalue is $f^{-1}(r)$.

In order to get sharp bounds for the first non-zero eigenvalue $\tau_{1, \phi}$ of the eigenvalue problem (1.15) on the compact MMS $\left(N,\langle\cdot, \cdot\rangle, e^{-\phi} d v\right)$ with boundary $\partial N$, we need the following facts which have been proven in [3, 4$]$.

Lemma 2.2. ([3, Proposition 2.2]) Assume that $u$ is a smooth function on $N^{n+1}$, and other same notations have the same meaning as those at the end of Subsection 1.2. Then we have

$$
|\operatorname{Hess} u|^{2}+\operatorname{Ric}_{\phi}(\nabla u, \nabla u) \geq \frac{\left(\Delta_{\phi} u\right)^{2}}{K}+\operatorname{Ric}_{\phi}^{K}(\nabla u, \nabla u)
$$

for every $K>n+1$ or $K=n+1$ and $\phi$ is constant. Moreover, equality holds if and only if Hess $u=\frac{\Delta u}{n+1}\langle\cdot, \cdot\rangle$ and $\langle\nabla u, \nabla \phi\rangle=-\frac{K-n-1}{K} \Delta_{\phi} u$.

By directly applying Lemma 2.2 and the generalized Reilly formula shown in [15], one can obtain the following fact.

Lemma 2.3. Assume that $u$ is a smooth function on $N^{n+1}, h=\frac{\partial u}{\partial \vec{\eta}}, z=\left.u\right|_{\partial N}, \bar{\nabla}$ is the gradient operator on $\partial N$, and other same notations have the same meaning as those at the end of Subsection 1.2. Then we have

$$
\int_{N} \frac{K-1}{K}\left[\left(\Delta_{\phi} u\right)^{2}-\operatorname{Ric}_{\phi}^{K}(\nabla u, \nabla u)\right] \geq \int_{\partial N}\left[2 h \bar{\Delta}_{\phi} z+n H_{\phi} h^{2}+I I(\bar{\nabla} z, \bar{\nabla} z)\right]
$$

for every $K>n+1$ or $K=n+1$ and $\phi$ is constant. Moreover, equality holds if and only if $\operatorname{Hess} u=\frac{\Delta u}{n+1}\langle\cdot, \cdot\rangle$ and $\langle\nabla u, \nabla \phi\rangle=-\frac{K-n-1}{K} \Delta_{\phi} u$.

Remark 2.4. The first conclusion of Lemma 2.3 is actually (2.5) in [⿰月]. 
Batista and Santos have proved the following conclusion, which is a slight modification to [18, Theorem 1.6].

Lemma 2.5. ([4, Proposition 2.2]) Let $N^{n+1}$ be a compact weighted Riemannian manifold with nonempty boundary $\partial N$ and $\operatorname{Ric}_{\phi}^{K} \geq 0$. If the second fundamental form of $\partial N$ satisfies $I I \geq c \mathrm{I}_{n \times n}$, in the quadratic form sense, and $H_{\phi} \geq \frac{K-1}{n} c$, then

$$
\lambda_{1}^{c}(\partial N) \geq(K-1) c^{2}
$$

where $\lambda_{1}^{c}$ is the first non-zero closed eigenvalue of the drifting Laplacian acting on functions on $\partial N$. The equality holds if and only if $N^{n+1}$ is isometric to a Euclidean ball of radius $\frac{1}{c}, \phi$ is constant and $K=n+1$.

\section{Proofs of the Steklov eigenvalue comparisons}

In this section, with the help of results mentioned in Section 2, by using a similar method to that shown by Escobar [13], we can give the proofs of Theorems 1.5 and 1.6 as follows.

Proof of Theorem 1.6. Let $\psi(t) e(\xi)$ be the eigenfunction for the first non-zero eigenvalue $v_{1}\left(\mathscr{B}_{n}\left(p^{+}, r\right)\right)$ on $\mathscr{B}_{n}\left(p^{+}, r\right)$ given as Fact 2 . By Fact 2 also, we have

$$
\psi^{\prime}(t)=\frac{n-1}{f^{n-1}(t)} \int_{0}^{t} \psi(s) f^{n-3}(s) d s
$$

which implies that $\psi$ is positive (resp., negative) in $(0, r)$ if $\psi^{\prime}(t)$ is positive (resp., negative) in a neighborhood of zero. So, the function $\psi$ determined in Fact 2 does not change sign in $(0, r)$. Without loss of generality, we can assume $\psi \geq 0$. Correspondingly, $\psi^{\prime}(t)>0$.

Consider the test function $\varphi(t, \xi)=a_{+}(t) e_{1}(\xi)$, where $e_{1}(\xi)$ is an eigenfunction of the first non-zero closed eigenvalue $\lambda_{1}^{c}(\partial B(p, r))$ of the Laplacian on the boundary $\partial B(p, r)$, and

$$
\begin{gathered}
a_{+}(t):=\max \{a(t), 0\} \\
a(t):=\psi(t)\left[\frac{f^{n-1}(t)}{h(t)}\right]^{1 / 2}+\int_{t}^{r} \psi(s)\left(\left[\frac{f^{n-1}(s)}{h(s)}\right]^{1 / 2}\right)^{\prime} d s,
\end{gathered}
$$

with $h(t):=\max \left\{d^{*}(t), \frac{f^{2}(t)}{n-1} d^{\sharp}(t)\right\}$ and

$$
\begin{gathered}
d^{\sharp}(t)=\int_{\mathbb{S}^{n-1}}\left|\nabla e_{1}\right|_{\mathbb{S}^{n-1}}^{2}(\xi) J^{n-3}(t, \xi), \\
d^{*}(t)=\int_{\mathbb{S}^{n-1}} e_{1}^{2}(\xi) \cdot \operatorname{det} \mathbb{A}(t, \xi)=\int_{\mathbb{S}^{n-1}} e_{1}^{2}(\xi) \sqrt{|g|}(t, \xi)=\int_{\mathbb{S}^{n-1}} e_{1}^{2}(\xi) J^{n-1}(t, \xi) .
\end{gathered}
$$

Clearly, $h(t)$ is Lipschitz continuous and hence differentiable almost everywhere. Besides, by Rayleigh's theorem and Max-min principle, we have

$$
\int_{\partial B(p, r)}\left|\nabla e_{1}\right|^{2}=\lambda_{1}^{c}(\partial B(p, r)) \cdot \int_{\partial B(p, r)} e_{1}^{2}
$$


and

$$
\int_{\partial B(p, r)} e_{1}(\xi)=0
$$

By Theorem 2.1, we have

$$
\left(\frac{\sqrt{|g|}}{f^{n-1}}\right)^{\prime}=\frac{(n-1) J^{n-2}}{f^{n}}\left(J^{\prime} f-f^{\prime} J\right) \geq 0
$$

in $(0, r)$, which implies

$$
f^{\prime} J-J^{\prime} f \leq 0
$$

in $(0, r)$.

Now, we claim that

$$
\left[\frac{f^{n-1}(t)}{h(t)}\right]^{\prime} \leq 0
$$

in $(0, r)$. This is because if $h(t)=d^{*}(t)$ in a neighborhood of some point in $(0, r)$, then together with (3.3), we have $h^{2}(t)\left[\frac{f^{n-1}(t)}{h(t)}\right]^{\prime}=(n-1) d^{*} f^{n-2} f^{\prime}-f^{n-1}(t) \cdot\left(d^{*}\right)^{\prime}=(n-1) f^{n-2} \int_{\mathbb{S}^{n-1}} e_{1}^{2} J^{n-2}\left(f^{\prime} J-J^{\prime} f\right) \leq 0$.

If $h(t)=\frac{f^{2}(t)}{n-1} d^{\sharp}(t)$ in a neighborhood of some point in $(0, r)$, then together with $(3.3)$, we have

$$
\begin{aligned}
\left(d^{\sharp}(t)\right)^{2} \cdot\left[\frac{f^{n-3}(t)}{d^{\sharp}(t)}\right]^{\prime} & =(n-3) d^{\sharp} f^{n-4} f^{\prime}-f^{n-3}(t) \cdot\left(d^{\sharp}\right)^{\prime} \\
& =(n-3) f^{n-4} \int_{\mathbb{S}^{n-1}}\left|\nabla e_{1}\right|_{\mathbb{S}^{n-1}}^{2} J^{n-4}\left(f^{\prime} J-J^{\prime} f\right) \leq 0,
\end{aligned}
$$

which implies $\left[\frac{f^{n-1}(t)}{h(t)}\right]^{\prime} \leq 0$. Therefore, our claim (3.4) is true. So, the function

$$
\int_{t}^{r} \psi(s)\left(\left[\frac{f^{n-1}(s)}{h(s)}\right]^{1 / 2}\right)^{\prime} d s
$$

is negative for $t<r$, except when $J(t, \xi)=f(t)$. Together with the definitions of $a(t)$ and $a_{+}(t)$, it follows that

$$
a(t) \leq a_{+}(t) \leq \psi(t)\left[\frac{f^{n-1}(t)}{h(t)}\right]^{1 / 2}
$$

on $(0, r)$. Using the above inequality, definitions of $h(t)$ and $d^{\sharp}(t)$, one can get

$$
\begin{aligned}
\int_{B(p, r)} a_{+}^{2}(t)\left|\nabla e_{1}\right|_{\mathbb{S}^{n-1}}^{2} J^{n-3}(t, \xi) d t d \sigma & =\int_{0}^{r} a_{+}^{2}(t)\left(\int_{\mathbb{S}^{n-1}}\left|\nabla e_{1}\right|_{\mathbb{S}^{n-1}}^{2} J^{n-3}(t, \xi) d \sigma\right) d t \\
& \leq \int_{0}^{r} \psi^{2} \frac{f^{n-1}(t) d^{\sharp}(t)}{h(t)} d t \\
& \leq(n-1) \int_{0}^{r} \psi^{2} f^{n-3}(t) d t,
\end{aligned}
$$


where, as before, $d \sigma$ denotes the $(n-1)$-dimensional volume element on $\mathbb{S}^{n-1}$. Therefore, we have

$$
\begin{aligned}
\int_{B(p, r)}|\nabla \varphi|^{2}= & \int_{B(p, r)}\left(a_{+}^{\prime}(t)\right)^{2} e_{1}^{2}(\xi) J^{n-1}(t, \xi) d t d \sigma \\
& \quad+\int_{B(p, r)} a_{+}^{2}(t)\left|\nabla e_{1}\right|_{\mathbb{S}^{n-1}}^{2} J^{n-3}(t, \xi) d t d \sigma \\
\leq & \int_{B(p, r)}\left(\psi^{\prime}(t)\right)^{2} \frac{f^{n-1}(t)}{h(t)} e_{1}^{2}(\xi) J^{n-1}(t, \xi) d t d \sigma+(n-1) \int_{0}^{r} \psi^{2} f^{n-3}(t) d t \\
= & \int_{0}^{r}\left(\psi^{\prime}(t)\right)^{2}\left(\frac{\int_{\mathbb{S}^{n-1}} e_{1}^{2}(\xi) J^{n-1}(t, \xi) d \sigma}{h(t)}\right) f^{n-1}(t) d t+(n-1) \int_{0}^{r} \psi^{2} f^{n-3}(t) d t \\
\leq & \int_{0}^{r}\left(\psi^{\prime}(t)\right)^{2} f^{n-1}(t) d t+(n-1) \int_{0}^{r} \psi^{2} f^{n-3}(t) d t .
\end{aligned}
$$

On the other hand, by direct calculation, we have

$$
\int_{\partial B(p, r)} \varphi^{2}=a_{+}^{2}(r) \int_{\mathbb{S}^{n-1}} e_{1}^{2}(\xi) J^{n-1}(r, \xi) d \sigma=\frac{\psi^{2}(r) f^{n-1}(r) d^{*}(r)}{h(r)} .
$$

Equality (3.1) is equivalent to

$$
\lambda_{1}^{c}(\partial B(p, r))=\frac{d^{\sharp}(r)}{d^{*}(r)} .
$$

Together with the assumption (1.11), we have

$$
d^{\sharp}(r) \leq d^{*}(r) \frac{n-1}{f^{2}(r)},
$$

which means $\frac{f^{2}(t)}{n-1} d^{\sharp}(t) \leq d^{*}(r)$. So, one has $h(r)=d^{*}(r)$, and then (3.6) becomes

$$
\int_{\partial B(p, r)} \varphi^{2}=\psi^{2}(r) f^{n-1}(r) .
$$

Combining (3.5) and (3.7) yields

$$
\frac{\int_{B(p, r)}|\nabla \varphi|^{2}}{\int_{\partial B(p, r)} \varphi^{2}} \leq \frac{\int_{0}^{r}\left(\psi^{\prime}(t)\right)^{2} f^{n-1}(t) d t+(n-1) \int_{0}^{r} \psi^{2} f^{n-3}(t) d t}{\psi^{2}(r) f^{n-1}(r)} .
$$

Since by (3.2), the equality

$$
\int_{\partial B(p, r)} \varphi=\psi(r) \int_{\partial B(p, r)} e_{1}(\xi)=0
$$

holds, and then by applying Fact 2, the characterization (1.2) and (3.8), we can obtain $v_{1}(B(p, r)) \leq \frac{\int_{B(p, r)}|\nabla \varphi|^{2}}{\int_{\partial B(p, r)} \varphi^{2}} \leq \frac{\int_{0}^{r}\left(\psi^{\prime}(t)\right)^{2} f^{n-1}(t) d t+(n-1) \int_{0}^{r} \psi^{2} f^{n-3}(t) d t}{\psi^{2}(r) f^{n-1}(r)}=v_{1}\left(\mathscr{B}_{n}\left(p^{+}, r\right)\right)$, 
which is exactly (1.12). It is easy to find that the equality in the above inequality holds if and only if $f(t)=J(t, \xi)$, which, by Theorem 2.1, Definition 1.4 and Fact 1, is equivalent to say that $B(p, r)$ is isometric to $\mathscr{B}_{n}\left(p^{+}, r\right)$. This completes the proof.

Now, in order to prove Theorem 1.5, we only need to confirm that the precondition (1.11) holds naturally for the case of two and three dimensions.

Proof of Theorem 1.5. We divide the proof into two cases as follows:

Case 1. If $n=2$, i.e., $M$ is a 2-dimensional Riemannian surface, then $\partial B(p, r)$ is connected and diffeomorphic to $\mathbb{S}^{1}$ because $r<\operatorname{inj}(p)$. Therefore, by Weinstock's Theorem [26], we have

$$
v_{1}(B(p, r)) \leq \frac{2 \pi}{L}=\frac{2 \pi}{\int_{0}^{2 \pi} J(t, \xi) d \sigma} .
$$

Here $L$ represents the perimeter of the closed curve $\partial B(p, r)$, which is diffeomorphic to $\mathbb{S}^{1}$. Applying Theorem 2.1. from which one has $J(t, \xi) \geq f(t)$ on $(0, r)$, and Fact 3 to (3.9), we can get

$$
v_{1}(B(p, r)) \leq \frac{2 \pi}{\int_{0}^{2 \pi} J(t, \xi) d \sigma} \leq \frac{2 \pi}{\int_{0}^{2 \pi} f(t) d \sigma}=\frac{1}{f(t)}=v_{1}\left(\mathscr{B}_{n}\left(p^{+}, r\right)\right) .
$$

Obviously, equality in the above inequality holds if and only if $f(t)=J(t, \xi)$, which, by Theorem 2.1, Definition 1.4 and Fact 1, is equivalent to say that $B(p, r)$ is isometric to $\mathscr{B}_{n}\left(p^{+}, r\right)$.

Case 2. If $n=3$, i.e., $M$ is a Riemannian 3-manifold, then $\partial B(p, r)$ is connected and diffeomorphic to $\mathbb{S}^{2}$ because $r<\operatorname{inj}(p)$. Let $e$ be any eigenfunction of the first non-zero closed eigenvalue $n-1=2$ of the Laplacian on $\mathbb{S}^{2}$. Therefore, $e=\langle\zeta, x\rangle$, where $\zeta \in \mathbb{S}^{2}$, and $x$ represents Euclidean coordinates. By direct calculation, we have

$$
\begin{aligned}
\frac{\int_{\partial B(p, r)}|\nabla e|^{2}}{\int_{\partial B(p, r)} e^{2}} & =\frac{\int_{\mathbb{S}^{n-1}}|\nabla e|_{\mathbb{S}^{n-1}}^{2} J^{n-3}(r, \xi) d \sigma}{\int_{\mathbb{S}^{n-1}} e^{2} J^{n-1}(r, \xi) d \sigma} \\
& =\frac{\int_{\mathbb{S}^{2}}|\nabla e|_{\mathbb{S}^{2}}^{2} d \sigma}{\int_{\mathbb{S}^{2}} e^{2} J^{2}(r, \xi) d \sigma} \\
& \leq \frac{1}{f^{2}(r)} \frac{\int_{\mathbb{S}^{2}}|\nabla e|_{\mathbb{S}^{2}}^{2} d \sigma}{\int_{\mathbb{S}^{2}} e^{2} d \sigma} \\
& =\frac{2}{f^{2}(r)}=\lambda_{1}^{c}\left(\partial \mathscr{B}_{n}\left(p^{+}, r\right)\right),
\end{aligned}
$$

where the last inequality holds because $J(t, \xi) \geq f(t)$ on $(0, r)$. On the other hand, define $F: \mathbb{S}^{2} \rightarrow$ $\mathbb{R}^{2}$ by $F\left(e_{\zeta}\right)=\int_{\partial B(p, r)} e_{\zeta}=\int_{\mathbb{S}^{2}} e_{\zeta} J^{2}(r, \xi) d \sigma$, and then we know that there must exist some $e \in \mathbb{S}^{2}$ such that $F(e)=0$, i.e.,

$$
\int_{\partial B(p, r)} e=\int_{\mathbb{S}^{2}} e \cdot J^{2}(r, \xi) d \sigma
$$

This is because the function $F$ is continuous and $F\left(e_{\zeta}\right)=-F\left(-e_{\zeta}\right)$ by choosing antipodal points. By (3.11), Rayleigh's theorem and Max-min principle, one has

$$
\lambda_{1}^{c}(B(p, r)) \leq \frac{\int_{\partial B(p, r)}|\nabla e|^{2}}{\int_{\partial B(p, r)} e^{2}} .
$$


Combining (3.10) and (3.12) results in

$$
\lambda_{1}^{c}(B(p, r)) \leq \lambda_{1}^{c}\left(\partial \mathscr{B}_{n}\left(p^{+}, r\right)\right)
$$

and then by applying Theorem 1.6 directly, we have $v_{1}(B(p, r)) \leq v_{1}\left(\mathscr{B}_{n}\left(p^{+}, r\right)\right)$. Clearly, the rigidity conclusion for the equality $v_{1}(B(p, r))=v_{1}\left(\mathscr{B}_{n}\left(p^{+}, r\right)\right)$ here can be attained by using almost the same argument as the 2-dimensional case.

This completes the proof.

Remark 3.1. It is easy to check that the method used in Case 2 here is also valid for Case 1.

\section{Some eigenvalue estimates}

Estimates for the first non-zero eigenvalue $\tau_{1, \phi}$ of the eigenvalue problem (1.15) with $\beta \geq 0$ will be given in this section. In fact, we can obtain the followings.

Theorem 4.1. Assume that $\left(N,\langle\cdot, \cdot\rangle, e^{-\phi} d v\right)$ is an $(n+1)$-dimensional compact connected MMS with smooth boundary $\partial N$. If $\operatorname{Ric}_{\phi}^{K} \geq 0$ and $H_{\phi} \geq \frac{(K-1) c}{n}, I I \geq c \cdot \mathrm{I}_{n \times n}$ for some positive constant $c>0$, with II the second fundamental form of $\partial N$, then, for the eigenvalue problem (1.15) with $\beta \geq 0$, we have

$$
\tau_{1, \phi} \leq \beta \lambda_{1}^{c}+\frac{\sqrt{\lambda_{1}^{c}}\left[\sqrt{\lambda_{1}^{c}}+\sqrt{\lambda_{1}^{c}-(K-1) c^{2}}\right]}{(K-1) c}
$$

where, similar as before, $\mathrm{I}_{n \times n}$ represents the $n \times n$ identity matrix, and $\lambda_{1}^{c}$ is the first non-zero closed eigenvalue of the drifting Laplacian on the boundary $\partial N$. Equality in (4.1) holds if and only if $N$ is isometric to an $(n+1)$-dimensional Euclidean ball of radius $\frac{1}{c}, \phi$ is the non-zero constant function, and $K=n+1$.

Proof. Let $u$ be the solution to the following problem

$$
\begin{cases}\Delta_{\phi} u=0 & \text { in } N \\ u=z & \text { on } \partial N\end{cases}
$$

where $z$ is the eigenfunction of the first non-zero closed eigenvalue $\lambda_{1}^{c}$ of the drifting Laplacian $\bar{\Delta}_{\phi}$ on $\partial N$. That is, $\bar{\Delta}_{\phi} z+\lambda_{1}^{c} z=0$ on $\partial N, \int_{\partial N} z=0$. Set $h=\frac{\partial u}{\partial \vec{\eta}}$. By (1.17) and the fact that $\int_{\partial N} z=\int_{\partial N} u=0$, we have

$$
\begin{aligned}
\tau_{1, \phi} & \leq \frac{\int_{N}|\nabla u|^{2}+\beta \int_{\partial N}|\bar{\nabla} z|^{2}}{\int_{\partial N} z^{2}} \\
& =\frac{\int_{\partial N} z h+\beta \int_{\partial N}|\bar{\nabla} z|^{2}}{\int_{\partial N} z^{2}} \\
& =\beta \lambda_{1}^{c}+\frac{\int_{\partial N} z h}{\int_{\partial N} z^{2}}
\end{aligned}
$$


where the first equality holds by the usage of the divergence theorem. By Lemmas 2.2 and 2.3, together with assumptions $\operatorname{Ric}_{\phi}^{K} \geq 0, H_{\phi} \geq \frac{(K-1) c}{n}, I I \geq c \cdot \mathrm{I}_{n \times n}$ for some positive constant $c>0$, we have

$$
\begin{aligned}
0 & \geq \int_{N} \frac{K-1}{K}\left[\left(\Delta_{\phi} u\right)^{2}-\operatorname{Ric}_{\phi}^{K}(\nabla u, \nabla u)\right] \\
& \geq \int_{\partial N}\left[n H_{\phi} h^{2}+2 h \bar{\Delta}_{\phi} z+I I(\bar{\nabla} z, \bar{\nabla} z)\right] \\
& \geq \int_{\partial N}\left[(K-1) c h^{2}-2 \lambda_{1}^{c} z h+c|\bar{\nabla} z|^{2}\right] \\
& =\int_{\partial N}\left[(K-1) c h^{2}-2 \lambda_{1}^{c} z h+c \lambda_{1}^{c} z^{2}\right],
\end{aligned}
$$

and then, by applying Hölder's inequality to the above inequality, it follows that

$$
\begin{aligned}
0 & \geq \int_{\partial N}\left[(K-1) c h^{2}-2 \lambda_{1}^{c} h z+c \lambda_{1}^{c} z^{2}\right] \\
& \geq \int_{\partial N}(K-1) c h^{2}-2 \lambda_{1}^{c}\left(\int_{\partial N} h^{2}\right)^{\frac{1}{2}}\left(\int_{\partial N} z^{2}\right)^{\frac{1}{2}}+\int_{\partial N} c \lambda_{1}^{c} z^{2} \\
& =(K-1) c\left[\left(\int_{\partial N} h^{2}\right)^{\frac{1}{2}}-\frac{\lambda_{1}^{c}}{(K-1) c}\left(\int_{\partial N} z^{2}\right)^{\frac{1}{2}}\right]^{2}+\left[c \lambda_{1}^{c}-\frac{\left(\lambda_{1}^{c}\right)^{2}}{(K-1) c}\right] \int_{\partial N} z^{2},
\end{aligned}
$$

which is equivalent with

$$
\left(\int_{\partial N} h^{2}\right)^{\frac{1}{2}} \leq \frac{\sqrt{\lambda_{1}^{c}}\left[\sqrt{\lambda_{1}^{c}}+\sqrt{\lambda_{1}^{c}-(K-1) c^{2}}\right]}{(K-1) c}\left(\int_{\partial N} z^{2}\right)^{\frac{1}{2}}
$$

Combining (4.2) and (4.3) yields

$$
\begin{aligned}
\tau_{1, \phi} & \leq \beta \lambda_{1}^{c}+\frac{\int_{\partial N} z h}{\int_{N} z^{2}} \\
& \leq \beta \lambda_{1}^{c}+\frac{\left(\int_{\partial N} h^{2}\right)^{\frac{1}{2}}}{\left(\int_{\partial N} z^{2}\right)^{\frac{1}{2}}} \\
& \leq \beta \lambda_{1}^{c}+\frac{\sqrt{\lambda_{1}^{c}}\left[\sqrt{\lambda_{1}^{c}}+\sqrt{\lambda_{1}^{c}-(K-1) c^{2}}\right]}{(K-1) c}
\end{aligned}
$$

which is exactly (4.1). If equality holds in (4.1), then all inequalities become equalities, and through the above argument, one has Hess $u=0$ and

$$
h=\frac{\sqrt{\lambda_{1}^{c}}\left[\sqrt{\lambda_{1}^{c}}+\sqrt{\lambda_{1}^{c}-(K-1) c^{2}}\right]}{(K-1) c} z
$$

Therefore, taking a local orthonormal fields $\left\{e_{i}\right\}_{i=1}^{n}$ tangent to $\partial N$, similar to the calculation in [ 4 , 
page 9], we can obtain

$$
\begin{aligned}
0 & =\sum_{i=1}^{n} \operatorname{Hess} u\left(e_{i}, e_{i}\right) \\
& =\sum_{i=1}^{n}\left\langle\nabla_{e_{i}} \nabla u, e_{i}\right\rangle \\
& =\bar{\Delta}_{\phi} z+n H_{\phi} h \\
& =-\lambda_{1}^{c} z+c(K-1) h \\
& =-\lambda_{1}^{c} z+c(K-1) \frac{\sqrt{\lambda_{1}^{c}}\left[\sqrt{\lambda_{1}^{c}}+\sqrt{\lambda_{1}^{c}-(K-1) c^{2}}\right]}{(K-1) c} z
\end{aligned}
$$

which implies $\lambda_{1}^{c}=(K-1) c^{2}$. Then, under the assumptions for $\operatorname{Ric}_{\phi}^{K}, I I$ and $H_{\phi}$, by Lemma 2.5, we know that $N$ is isometric to an $(n+1)$-dimensional Euclidean ball of radius $\frac{1}{c}, \phi$ is a non-zero constant function and $K=n+1$. This completes the proof.

A lower bound for $\tau_{1, \phi}$ can also be obtained as follows.

Theorem 4.2. Assume that $\left(N,\langle\cdot, \cdot\rangle, e^{-\phi} d v\right)$ is an $(n+1)$-dimensional compact connected MMS with smooth boundary $\partial N$. If $\operatorname{Ric}_{\phi}^{K} \geq 0, H_{\phi} \geq \frac{(K-1) c}{n}, I I \geq c \cdot \mathrm{I}_{n \times n}$ for some positive constant $c>0$, then, for the eigenvalue problem (1.15) with $\beta \geq 0$, we have

$$
\tau_{1, \phi}>\frac{1}{2} c\left[1+(K-1) c \beta+\sqrt{(K-1) c^{2} \beta^{2}+2(K-1) c \beta}\right] .
$$

Proof. We use a similar proof to that of [27, Theorem 1.3]. In order to state precisely, we divide the proof into two cases as follows:

Case 1. Assume that $\beta=0$. Let $u$ be an eigenfunction of the first non-zero eigenvalue $\tau_{1, \phi}$ of the eigenvalue problem (1.15) with $\beta=0$. Set $h=\left.\frac{\partial u}{\partial \vec{\eta}}\right|_{\partial N}, z=\left.u\right|_{\partial N}$. By Lemmas 2.2 and 2.3, together with the assumptions $\operatorname{Ric}_{\phi}^{K} \geq 0, H_{\phi} \geq \frac{(K-1) c}{n}, I I \geq c \cdot \mathrm{I}_{n \times n}$ for some positive constant $c>0$, we have

$$
\begin{aligned}
0 & \geq \int_{\partial N} \frac{K-1}{K}\left[\left(\Delta_{\phi} u\right)^{2}-\operatorname{Ric}_{\phi}^{K}(\nabla u, \nabla u)\right] \\
& \geq \int_{\partial N}\left[n H_{\phi} h^{2}+2 h \bar{\Delta}_{\phi} z+I I(\bar{\nabla} z, \bar{\nabla} z)\right] \\
& >\int_{\partial N}[-2\langle\bar{\nabla} z, \bar{\nabla} h\rangle+c\langle\bar{\nabla} z, \bar{\nabla} z\rangle] \\
& =\int_{\partial N}\left[-2 \tau_{1, \phi}\langle\bar{\nabla} z, \bar{\nabla} z\rangle+c\langle\bar{\nabla} z, \bar{\nabla} z\rangle\right]
\end{aligned}
$$

which implies

$$
\tau_{1, \phi}>\frac{c}{2} .
$$


Case 2. Assume that $\beta>0$. Let $u$ be an eigenfunction of the first non-zero eigenvalue $\tau_{1, \phi}$ of the eigenvalue problem (1.15) with $\beta>0$. Set $\gamma=\frac{1}{\beta}, \tau=\frac{\tau_{1, \phi}}{\beta}, h=\left.\frac{\partial u}{\partial \vec{\eta}}\right|_{\partial N}, z=\left.u\right|_{\partial N}$. By (1.15), one has

$$
\Delta_{\phi} u=0, \quad \bar{\Delta}_{\phi} z=\gamma h-\tau z
$$

By Lemmas 2.2 and 2.3, together with the assumption $\operatorname{Ric}_{\phi}^{K} \geq 0$, we have

$$
0 \geq \int_{N} \frac{K-1}{K}\left[\left(\Delta_{\phi} u\right)^{2}-\operatorname{Ric}_{\phi}^{K}(\nabla u, \nabla u)\right] \geq \int_{\partial N}\left[n H_{\phi} h^{2}+2 h \bar{\Delta}_{\phi} z+I I(\bar{\nabla} z, \bar{\nabla} z)\right]
$$

which, together with assumptions $H_{\phi} \geq \frac{(K-1) c}{n}, I I \geq c \cdot \mathrm{I}_{n \times n}$ for some positive constant $c>0$, implies

$$
\begin{aligned}
0 & \geq \int_{\partial N}\left[(K-1) c h^{2}+2 h(\gamma h-\tau z)+c|\bar{\nabla} z|^{2}\right] \\
& =\int_{\partial N}\left[(K-1) c h^{2}+2 \gamma h^{2}-2 \tau h z-c z \bar{\Delta}_{\phi} z\right] \\
& =\int_{\partial N}\left[c \tau z^{2}-(2 \tau+c \gamma) h z+((K-1) c+2 \gamma) h^{2}\right] \\
& =[(K-1) c+2 \gamma] \int_{\partial N}\left[h-\frac{\left(\tau+\frac{c \gamma}{2}\right) z}{(K-1) c+2 \gamma}\right]^{2}+\left[c \tau-\frac{\left(\tau+\frac{c \gamma}{2}\right)^{2}}{(K-1) c+2 \gamma}\right] \int_{\partial N} z^{2}
\end{aligned}
$$

Therefore, from the above inequality, one has

$$
\left[\frac{\left(\tau+\frac{c \gamma}{2}\right)^{2}}{(K-1) c+2 \gamma}-c \tau\right] \int_{\partial N} z^{2} \geq[(K-1) c+2 \gamma] \int_{\partial N}\left[h-\frac{\left(\tau+\frac{c \gamma}{2}\right) z}{(K-1) c+2 \gamma}\right]^{2},
$$

which implies

$$
\frac{\left(\tau+\frac{c \gamma}{2}\right)^{2}}{(K-1) c+2 \gamma}-c \tau \geq 0
$$

Solving the above inequality yields

$$
\tau \geq \frac{\left[\sqrt{(K-1)^{2} c^{2}+2 c(K-1) \gamma}+\gamma+(K-1) c\right] c}{2}
$$

or

$$
\tau \leq \frac{\left[\gamma+(K-1) c-\sqrt{(K-1)^{2} c^{2}+2 c(K-1) \gamma}\right] c}{2} .
$$

Now, we show that (4.10) cannot happen. In fact, multiplying the second equation of (4.6) by $z$ and integrating over $\partial N$, one can obtain

$$
\begin{aligned}
\tau \int_{\partial N} z^{2} & =\int_{\partial N}|\bar{\nabla} z|^{2}+\gamma \int_{\partial N} h z \\
& =\int_{\partial N}|\bar{\nabla} z|^{2}+\gamma \int_{N}|\nabla u|^{2}
\end{aligned}
$$


where the fact $\Delta_{\phi} u=0$ in $N$ has been used. Since $\int_{\partial N} z=0, z \neq 0$, by Lemma 2.5, it follows that

$$
\int_{\partial N}|\bar{\nabla} z|^{2} \geq \lambda_{1}^{c}(\partial N) \int_{\partial N} z^{2} \geq(K-1) c^{2} \int_{\partial N} z^{2} .
$$

Combining (4.11) and (4.12), together with the fact $\int_{N}|\nabla u|^{2}>\frac{c}{2} \int_{\partial N} z^{2}$, yields

$$
\tau>(K-1) c^{2}+\frac{c \gamma}{2}
$$

which implies that (4.10) cannot hold. In order to get the estimate (4.4), one only needs to exclude the equality case in (4.9). We shall get this fact by contradiction. Suppose that

$$
\tau=\frac{\left[\sqrt{(K-1)^{2} c^{2}+2 c(K-1) \gamma}+\gamma+(K-1) c\right] c}{2} .
$$

From the above argument, we know that if (4.13) holds, then all inequalities in (4.7) and (4.8) should take equality sign. Therefore, it follows that

$$
h=\frac{\left(\tau+\frac{c \gamma}{2}\right) z}{(K-1) c+2 \gamma} \quad \text { on } \partial N,
$$

$I I=c \mathrm{I}_{n \times n}$, i.e., all principal curvatures of $\partial N$ equal $c$, and, by Lemma 2.3 and the first equation of (4.6),

$$
\text { Hess } u=\frac{\Delta_{\phi} u-\frac{K-n-1}{K} \Delta_{\phi} u}{n+1}\langle\cdot, \cdot\rangle=0 \quad \text { on } N .
$$

Using the restriction of (4.15) on the boundary, i.e., Hess $z=0$ on $\partial N$, and $I I=c \mathrm{I}_{n \times n}$, one can get

$$
h=c z
$$

Combining (4.14) and (4.16) yields

$$
\frac{\tau+\frac{c \gamma}{2}}{(K-1) c+2 \gamma}=c
$$

which, together with (4.13), implies $\gamma=0$. This is a contradiction. Hence, we have

$$
\tau>\frac{\left[\sqrt{(K-1)^{2} c^{2}+2 c(K-1) \gamma}+\gamma+(K-1) c\right] c}{2},
$$

which implies (4.4). Besides, when $\beta=0$, (4.5) is equivalent with (4.4). Summing up Cases 1 and 2 , our estimate (4.4) can be achieved for the eigenvalue problem (1.15) with $\beta \geq 0$.

Remark 4.3. When $\beta=0$, our estimate (4.1) is exactly the main estimate (1.6) in [4], which means Theorem 4.1] here covers [甘], Theorem 1.1] as a special case. When $\beta=0$ and $\phi=$ const. is a non-zero constant function, the Wentzell eigenvalue problem (1.15) of the weighted Laplacian degenerates into the classical Steklov eigenvalue problem (1.1), and naturally $\tau_{1, \phi}=v_{1}$, i.e., the first non-zero Steklov eigenvalue. In this setting, by Theorem 4.2, one has $v_{1}>\frac{c}{2}$, which is exactly Escobar's estimate in [11, Theorem 8]. That is to say, Theorem 4.2 here covers Escobar's conclusion [11, Theorem 8] as a special case. 


\section{An open problem}

If one checks Section 3 carefully, then naturally the following interesting problem would be proposed.

Open problem. For $n \geq 4$, is the Escobar-type Steklov eigenvalue inequality (1.12) also true without the precondition (1.11)?

\section{Acknowledgments}

This research was supported in part by the National Natural Science Foundation of China (Grant Nos. 11401131 and 11801496), China Scholarship Council, the Fok Ying-Tung Education Foundation (China), the NSF of Hubei Provincial Department of Education (Grant No. D20184301), and Hubei Key Laboratory of Applied Mathematics (Hubei University). The eigenvalue comparisons here for the Steklov eigenvalue problem have been already announced by Prof. Jing Mao in a talk at School of Mathematical Sciences, Fudan University in May, 2017. The corresponding author, Prof. J. Mao, wants to thank the Department of Mathematics, IST, University of Lisbon for its hospitality during his visit from September 2018 to September 2019.

\section{References}

[1] D. Bakry, M. Émery, Diffusion hypercontractives, Sém. Prob. XIX, Lect. Notes in Math. 1123 (1985) 177-206.

[2] M. F. Betta, F Brock, A. Mercaldo, M. R. Posterar, A weighted isoperimetric inequality and applications to symmetrization, J. Inequal. Appl. 4 (1999) 215-240.

[3] M. Batista, M. P. Cavalcante, J. Pyo., Some isoperimetric inequalities and eigenvalue estimates in weighted manifolds, J. Math. Anal. Appl. 419 (2014) 617-626.

[4] M. Batista, J. I. Santos, The first Stekloff eigenvalue in weighted Riemannian manifolds, available online at arXiv:1504.02630v1.

[5] A. P. Calderón, On an inverse boundary value problem, in "Seminar in Numerical Analysis and Its Applications to Continuum Physics", pp. 65-73, Soc. Brasileira de Matemática, Rio de Janeiro, 1980.

[6] S. Y. Cheng, Eigenvalue comparison theorems and its geometric application, Math. Z. 143 (1975) 289-297.

[7] S. Y. Cheng, Eigenfunctions and eigenvalues of the Laplacian, Am. Math. Soc. Proc. Symp. Pure Math. (Part II) 27 (1975) 289-297.

[8] M. Dambrine, D. Kateb, J. Lamboley, An extremal eigenvalue problem for the WentzellLaplace operator, Ann. I. H. Poincaré Non Linear Analysis 33(2) (2014) 409-450. 
[9] F. Du, Q. L. Wang, C. Y. Xia, Estimates for eigenvalues of the Wentzell-Laplace operator, Journal of Geometry and Physics 129 (2018) 25-33.

[10] F. Du, J. Mao, Q. L. Wang, C. Y. Xia, Isoperimetric bounds for eigenvalues of the WentzellLaplace, the Laplacian and a biharmonic Steklov problem, submitted and available online at arXiv:1808.10578.

[11] J. F. Escobar, The geometry of the first non-zero Stekloff eigenvalue, J. Funct. Anal. 150 (1997) 544-556.

[12] J. F. Escobar, An isoperimetric inequality and the first Steklov eigenvalue, J. Funct. Anal. 165 (1999) 101-116.

[13] J. F. Escobar, A comparison theorem for the first non-zero Steklov eigenvalue, J. Funct. Anal. 178 (2000) 143-155.

[14] L. B. Hou, J. Mao, The principle of numerical calculations for the eigenvalue comparison on parameterized surfaces, J. Math. Res. Appl. 38 (2018) 58-62.

[15] L. Ma, S. H. Du, Extension of Reilly formula with applications to eigenvalue estimates for drifting Laplacians, C. R. Math. Acad. Sci. Paris 348 (2010) 1203-1206.

[16] P. Freitas, J. Mao and I. Salavessa, Spherical symmetrization and the first eigenvalue of geodesic disks on manifolds, Calc. Var. Partial Differential Equations 51 (2014) 701-724.

[17] M. Gromov, Isopermetric of Waists and concentration of maps, Geom. Funct. Anal. 13(1) (2003) 178-215.

[18] Q. Huang, Q. H. Ruan, Application of some elliptic equations in Riemannian manifolds, J. Math. Anal. Appl. 409 (2014) 189-196.

[19] N. N. Katz and K. Kondo, Generalized space forms, Trans. Amer. Math. Soc. 354 (2002) 2279-2284.

[20] J. Mao, Eigenvalue estimation and some results on finite topological type, Ph.D. thesis, ISTUTL, 2013.

[21] J. Mao, Eigenvalue inequalities for the p-Laplacian on a Riemannian manifold and estimates for the heat kernel, J. Math. Pures Appl. 101(3) (2014) 372-393.

[22] J. Mao, F. Du, C. X. Wu, Eigenvalue Problems on Manifolds, Science Press, Beijing, 2017.

[23] B. O'Neill, Semi-Riemannian Geometry with applications to relativity, vol. 103 of Pure and Applied mathematics, Academic Press, San Diego, 1983.

[24] P. Petersen, Riemannian Geometry, Second Edition, vol.171 of Graduate Texts in Mathematics, Springer, New York, 2006.

[25] M. W. Stekloff, Sur les problèmes fondamentaux de la physique mathématique, Ann. Sci. École Norm. Sup. 19 (1902) 455-490. 
[26] R. Weinstock, Inequalities for a classical eigenvalue problem, J. Rational Mech. Anal. 3 (1954) 745-753.

[27] C. Y. Xia, Q. L. Wang, Eigenvalues of the Wentzell-Laplace operator and of the fourth order Steklov problems, Journal of Differential Equations 264(10) (2018) 6486-6506. 